\title{
Clinical presentations, radiological characteristics, and biological risk factors of cerebral venous thrombosis at a University Hospital in Saudi Arabia
}

Abdullah S. Alamri, MD, Mohammed F. Almuaigel, MD, Azra Zafar, MD, Foziah J. Alshamrani, MD, Noor M. AlMohish, MD, Mona H. AlSheikh, MD.

\begin{abstract}
الأهداف : خثار الوريد الدماغي هو نوع غير شائع نسبيًا من السكتة الدماغية

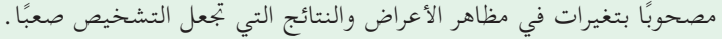

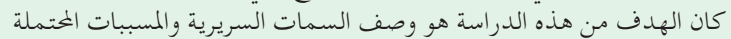
لخثار الوريد الدماغي في المملكة العربية السعودية وصني السيمات السرية

المنهجية : تم تنفيذ تصميم رصد بأثر رجعي . تم الحصول على وتحت وتحليل البيانات

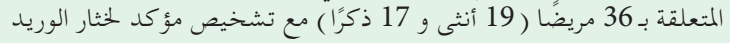

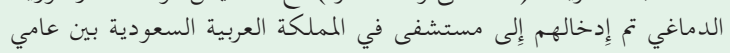

2019,2008

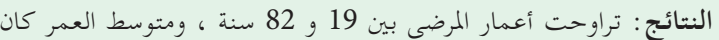

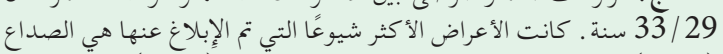

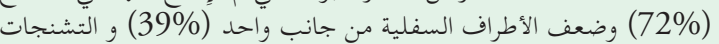

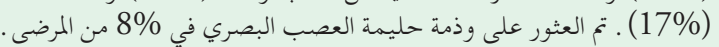

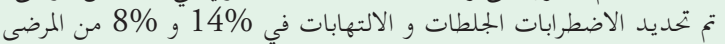

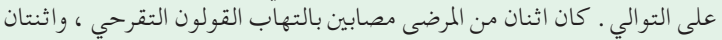

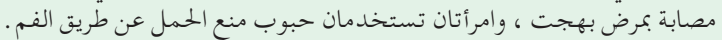

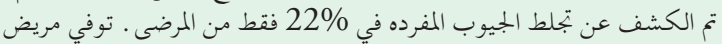

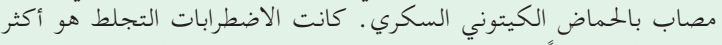

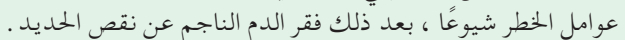

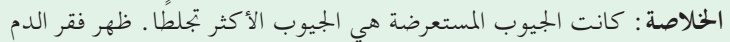

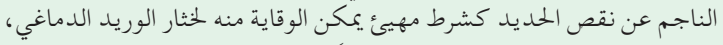

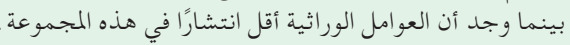

Objectives: To describe the clinical features and possible etiologies of cerebral vein thrombosis (CVT) in a Saudi Arabian cohort.

Methods: A retrospective, observational design was implemented. Data pertaining to 36 patients (19 female and 17 male) with confirmed CVT diagnosis admitted to a hospital in Saudi Arabia between 2008 and 2019 were obtained and analyzed.

Results: The age of patients ranged between 19 to 82 years, and the mean/median age was $33 / 29$ years. Most commonly reported symptoms were headache
(72\%), unilateral lower limb weakness (39\%), and seizures (17\%). Papilledema was found in $8 \%$ of patients. Thrombotic disorders were identified in $14 \%$ and infections were identified in $8 \%$ of the patients. Two patients had ulcerative colitis, 2 were diagnosed with Behcet's disease, and 2 women were using oral contraceptive pills. Single sinus thrombosis was detected in only $22 \%$ of patients. One patient with diabetic ketoacidosis died. Thrombotic disorder was the most common risk factor, followed by that iron deficiency anemia.

Conclusion: The transverse sinus was the most frequently thrombosed sinus. Iron deficiency anemia emerged as a predisposing preventable condition for CVT, while genetic factors were found to be less important in this cohort.

Keywords: cerebral vein thrombosis, cerebral venous sinus thrombosis, Saudi Arabia, stroke, young adults

Saudi Med J 2021; Vol. 42 (2): 213-218 doi: 10.15537/smj.2021.2.25667

From the Neurology Department (Alamri, Almuaigel, Alshamrani, Zafar, AlMohish), King Fahad University Hospital, Al Khobar; from the Neurology Department, College of Medicine (Alamri, Alshamrani, Zafar), Imam Abdulrahman Bin Faisal University; and from the Physiology Department (AlSheikh), College of Medicine, Imam Abdulrahman Bin Faisal University, Al Dammam, Kingdom of Saudi Arabia.

Received 14th October 2020. Accepted 21st December 2020.

Address correspondence and reprint request to: Dr. Mohammed F. Almuaigel, Neurology Department, King Fahad University Hospital, Al Khobar, Kingdom of Saudi Arabia. E-mail:al.muaigel@hotmail.com

ORCID ID: https://orcid.org/0000-0001-6161-8179 


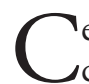
erebral venous sinus thrombosis (CVT) is considered one of the rare causes of stroke. It comprises $0.5-1 \%$ of all strokes and particularly affects younger patients. ${ }^{1}$ However, recent literature reported CVT as being more prevalent than mentioned previously, but still affecting less than 5 people per million. ${ }^{2}$ Furthermore, the number of cases reported in national cross-sections indicated a slightly varied and higher incidence of such cases. A recent study from Iran reported an estimated annual incidence of 13.5 cases per million. ${ }^{3}$ An estimated incidence of $<10$ cases per million per year was reported by Daif et $\mathrm{al}^{4}$ from Kingdom of Saudi Arabia (KSA). Yaqub et $\mathrm{al}^{5}$ has found CVT in $2 \%$ of all strokes. Due to the rarity of CVT demonstrated in these figures, information regarding the clinical presentation, course, and prognosis of CVT is limited and largely based on the studies with modest sample size. ${ }^{2}$ Nevertheless, the clinical manifestations are varied depending on the location of thrombus and this can often delay the diagnosis. Headache is the most commonly reported symptom (70-95\%). ${ }^{6,7}$ Seizures, focal neurological deficits, cranial nerve palsies, and altered mentation are other commonly reported features. $^{8}$

There are several known modifiable and nonmodifiable predisposing factors for CVT. Female gender, congenital or acquired thrombophilia, and infections are some of the common causative factors. ${ }^{9}$ Significantly higher prevalence among females has been attributed to the peripartum state and the use of oral contraceptive pills (OCPs). ${ }^{10}$ To make a timely diagnosis, physicians should have high index of suspicion. Nonenhanced computed tomography (NECT) is the most frequently performed neuroimaging technique in the emergency setting; however, direct signs of CVT are shown only in one-third of cases. ${ }^{11}$ Therefore, some diagnoses was initially missed. The prognosis is more favorable in CVT compared with arterial or hemorrhagic stroke. ${ }^{12}$ The list of predisposing or risk factors is long and include old age ( $>55$ years), oral contraception, hormone replacement therapy, obesity, pregnancy and puerperium, dehydration, infection, thrombophilic coagulopathy, local cerebral tumor, aneurysm or arteriovenous malformation. ${ }^{13-16}$ Despite significant advancements in diagnostic modalities and management strategies, CVT in KSA should be more investigated.

Disclosure. Authors have no conflict of interests, and the work was not supported or funded by any drug company.
The purpose of this study was to determine the frequency of various initial clinical symptoms, phenotypic spectra, risk factors, and radiological findings among patients diagnosed with CVT at the Neurology Department, King Fahd University Hospital, Al Khobar, KSA.

Methods. This retrospective observational study was carried out after the ethical approval was obtained from the Institutional Review Board of Imam Abdulrahman Bin Faisal University, Al Khobar, KSA. Informed consent was waived provided the retrospective nature of the study. Data was collected for patients admitted between January 2008 and December 2019. Data of all patients were maintained according to the International Statistical Classification of Diseases and Related Health Problems $\left(10^{\text {th }}\right.$ revision) system in an electronic data bank system of the hospital. Patients with ischemic stroke, hemorrhagic stroke, and transient ischemic attack were excluded. Finally, 36 patients with diagnosis of CVT were included in the study.

Diagnosis was based on clinical signs in patients presenting with features suggestive of a CVT, including headache, new onset seizures, focal neurological deficits, cranial nerve palsies, blurred vision, and alteration of consciousness level. Diagnosis was confirmed by brain and head CT scan, CT venogram (CTV), magnetic resonance imaging (MRI), MR venogram (MRV), and conventional 4 vessel cerebral angiogram. The MRI, MRA, and MRV were performed using a 1.5-Tesla MR imager including T1, T2, fluid attenuated inversion recovery imaging, diffusion-weighted imaging, apparent diffusion coefficient map, and gradient echo sequences. In order to analyze the frequency of predisposing factors, results of complete blood count, routine blood biochemistry, and coagulation profile were studied in all patients. In addition, results of hypercoagulable states including protein $\mathrm{C}$, protein $\mathrm{S}$, antithrombin III, factor V Leiden, homocysteine, antinuclear antibodies, anticardiolipin antibodies, antiphospholipid antibodies, lupus anticoagulant, and antibodies to double stranded DNA were also sought. Other investigations such as cerebrospinal fluid analysis were performed where required.

A structured preformat was designed that was comprised of details regarding demographic characteristics including age, gender, nationality, symptoms, and signs at time of presentation including headache, seizure, blurred vision, focal weakness or numbness, speech difficulty, cranial nerve palsies, and altered sensorium, blood investigations, and details of imaging modalities and radiological findings (location 
of thrombus, number of involved venous sinuses, venous infarction, and hemorrhagic transformation). Female patients were asked about pregnancy, puerperium and usage of oral contraceptive pills.

Statistical analysis. The analysis was performed using IBM SPSS Statistics for Windows, version 22 (IBM Corp, Armonk, NY, USA). Mean and standard deviation $( \pm S D)$ were computed for age and scaled data. Frequencies and percentages were calculated for categorical data.

Results. A total of 998 patients was diagnosed with acute stroke and 36 had confirmed CVT. Median age was 35 years for females and 26 for males with a female-to-male ratio of 1.1:1. The age ranged was from 19 to 82 years and the mean length of hospital stay was $18.4 \pm 15$ days. Three patients visited the hospital earlier with headache and dizziness, but were not admitted and were discharged after symptomatic treatment and later on developed severe headaches. Two patients developed hemiparesis while in the hospital and stroke code was activated for one patient. The most common presenting symptom was headache, followed by hemiparesis and seizures. Patient demographic characteristics and clinical features are detailed in Table 1.

Table 1 - Demographic characteristics and clinical features.

\begin{tabular}{lcc}
\hline Characteristics & $\mathbf{n}(\%)$ \\
\hline Age (Mean \pm SD years) & $33.5 \pm 12$ \\
Total & $28.3 \pm 7.2$ \\
Male & $37.5 \pm 15$ \\
Female & \\
Gender & $17(47.2)$ \\
Male & $19(52.7)$ \\
Female & \\
Clinical features & $26(72.2)$ \\
Headache & $14(38.8)$ \\
Hemiparesis & $7(19.4)$ \\
Vomiting & $6(16.6)$ \\
Seizures & $6(16.6)$ \\
Blurred vision & $5(13.8)$ \\
Altered sensorium & $7(19.4)$ \\
Papilledema & 2 & $(5.5)$ \\
Sixth nerve palsy & 1 & $(2.7)$ \\
Tinnitus & \\
\hline \multicolumn{2}{c}{ Data is presented as numbers and } \\
\multicolumn{2}{c}{ percentages $(\%)}$. & \\
\hline
\end{tabular}

The risk factors were divided into thrombotic disorders, female related factors, infections, and others. Details of risk factors have been summarized in Table 2. The most commonly identified risk factors was thrombotic disorder. Other risk factors were puerperium, usage of OCPs, infection, ulcerative colitis, Behcet's disease, and dehydration. Associated comorbidities were diabetes mellitus type 2 (4/36), and hypothyroidism (2/36). Cerebrospinal fluid analysis was carried out in 3 patients. Only one patient with a recurrent ear infection had abnormal parameters suggestive of acute bacterial meningitis. One patient with newly diagnosed diabetes mellitus was brought to the emergency department (ED) with complaints of dizziness and altered sensorium and while receiving treatment for diabetic ketoacidosis (DKA) developed hemiparesis. Stroke code was activated but CT of the brain showed hemorrhagic venous infarction and CTV showed non-visualization of right transverse and sigmoid sinuses. An emergent decompressive craniotomy was performed, but the patient did not survive.

Patterns of venous sinuses and veins involvement have been detailed in Table 3. Venous hemorrhagic infarction was detected in $13(36.1 \%)$, non-hemorrhagic infarction in $4(11.1 \%)$, and subarachnoid hemorrhage

Table 2 - Details of predisposing factors in patients with cerebral venous sinus thrombosis.

\begin{tabular}{lcc}
\hline Risk factors & $\mathbf{n}$ & $(\%)$ \\
\hline Infections & & \\
$\quad$ Mastoiditis & 2 & $(5.5)$ \\
Meningitis and cholesteatoma & 1 & $(2.7)$ \\
Female related factor & & \\
$\quad$ Puerperium & 5 & $(13.8)$ \\
Oral contraceptive usage & 4 & $(11.1)$ \\
Thrombotic disorders & & \\
Protein S deficiency & 5 & $(13.8)$ \\
Hyperhomocysteinemia & 1 & $(2.7)$ \\
Positive lupus anticoagulant & 4 & $(11.1)$ \\
Positive antiphospholipid and & 1 & $(2.7)$ \\
anticardiolipin antibodies & & \\
Polycythemia & 1 & $(2.7)$ \\
Other & & \\
Iron deficiency anemia & 11 & $(30.5)$ \\
Sickle cell trait & 3 & $(8.3)$ \\
Behcet's disease & 2 & $(5.5)$ \\
Ulcerative colitis & 2 & $(5.5)$ \\
Diabetic ketoacidosis & 1 & $(2.7)$ \\
Dehydration & 2 & $(5.5)$ \\
\hline & &
\end{tabular}


Table 3 - Number and pattern of venous sinus involvement

\begin{tabular}{lcc}
\hline Thrombosed venous sinuses and veins & $\mathbf{n}$ & $(\%)$ \\
\hline Transverse sinus & 28 & $(77.7)$ \\
Sigmoid sinus & 22 & $(61.1)$ \\
Internal jugular vein & 17 & $(47.2)$ \\
Superior sagittal sinus & 16 & $(44.4)$ \\
Straight sinus & 3 & $(8.3)$ \\
Vein of Galen & 2 & $(5.5)$ \\
Inferior sagittal sinus & 1 & $(2.7)$ \\
Single sinus involvement & & \\
$\quad$ Superior sagittal sinus alone & 5 & $(13.8)$ \\
$\quad$ Straight sinus alone & 1 & $(2.7)$ \\
Two sinuses involvement & & \\
$\quad$ Sigmoid and transverse sinus & 4 & $(11.1)$ \\
Transverse and superior sagittal sinus & 2 & $(5.5)$ \\
Transverse and straight sinus & 1 & $(2.7)$ \\
Sigmoid and cavernous sinus & 1 & $(2.7)$ \\
\hline
\end{tabular}

in $2(5.5 \%)$ out of 36 patients. The transverse sinus was the most commonly thrombosed sinus followed by the sigmoid sinus. Involvement of the internal jugular vein was more prevalent in females compared to males $(p=0.04)$. One patient with protein deficiency had severe thrombosis affecting all sinuses including the vein of Galen. The majority of patients had involvement of more than 2 sinuses. Hemorrhagic venous infarction was detected in $11(30.5 \%)$ patients. Hyperdense venous sinuses were seen in NECT of $6(16.6 \%)$ patients in our study and subarachnoid hemorrhage in 2 (5.5\%) patients.

Out of 36 patients, 35 were treated with anticoagulants and only one received antiplatelet drugs; 2 patients received endovascular treatment as well. The majority of patients $(86 \%)$ were discharged home with a modified rankin scale score of less than 3. Mortality in our cohort of patients was $2.7 \%$. Follow-up imaging was not available for all patients.

Discussion. Cerebral venous sinus thrombosis comprised $3.6 \%$ of all strokes in our study, which is higher than reported from Western countries and from KSA. ${ }^{1,5}$ Some studies, including one from KSA, have reported significant female preponderance, which was not observed in our study. ${ }^{11,14-16}$ However, studies from India also did not identify gender dissimilarity. ${ }^{10,17}$

Mean age in our cohort of patients was less than reported in other studies (38.3-54.4 years), and higher than that reported in studies from KSA.,16,19-23 However, there are studies which have reported mean ages comparable to those found in our study. ${ }^{11,24}$

The clinical features in patients with CVT are varied and depend not only on the extent and location of the thrombus, but also on patient age and duration of symptoms. ${ }^{2}$ Headache is considered the most frequent symptom of CVT, as it is found in nearly $90 \%$ of patients. ${ }^{14,25,26}$ It was found in $73 \%$ of our patients, and this was similar to the findings of Algahtani et $\mathrm{al}^{13}$ and Kajtazi et al. ${ }^{19}$ Two patients presented to the ED earlier with headaches and were discharged after symptomatic treatment without neuroimaging. One patient revisited the ED with increasing severity of symptoms and was found to have hyperdense sinuses on CT scan and diagnosis was confirmed by CTV and MRV. Another patient, who was 2 months postpartum, later on presented with hemiparesis and seizures. She was found to have left parietal and temporal hemorrhagic venous infarction on CT and thrombosed superior sagittal sinus, left transverse sinus, and sigmoid sinuses on CTV. These cases signify the importance of having a high index of suspicion for CVT in new onset headaches to prevent the delay in diagnosis and promote timely management. Approximately half of the cases develop focal neurological deficits as a result of hemorrhagic transformation of venous infarcts. ${ }^{14,28}$ Thirty-nine percent of our patients had hemiparesis, which is less than the $54.5 \%$ reported in another study from KSA. ${ }^{19}$ Daif et $\mathrm{al}^{4}$ reported focal abnormalities in only $32 \%$ of patients and related it to rapid diagnosis within 48 hours of the onset of symptoms. Focal neurological deficit was found in only $18.2 \%$ of patients in a recent multicenter study. ${ }^{26}$

Seizures were reported less frequently in our cohort of patients than mentioned in other studies (24\%-54.5\%). ${ }^{14,16,22}$ Altered sensorium was observed in $14 \%$, which is nearly the same as reported by Duman et $\mathrm{al}^{26}(17.8 \%)$ and Breteau et $\mathrm{al}^{29}(18.2 \%)$, but less than in some other studies (22\% to $50 \%) .{ }^{17,18}$ Relatively lower frequency of seizures and altered sensorium in our study could be related to the early presentation of patients.

Algahtani et $a{ }^{13}$ have reported the visual complaints of field defects and diminution of vision in 10.8\% $(12 / 111)$ of patients. However, it was higher in our study.

More than half of our patients had involvement of multiple sinuses (more than 2), which is in accordance with other studies. ${ }^{19,30}$ Duman et $\mathrm{al}^{26}$ has also observed involvement of multiple sinuses, example: more than 2 in $18 \%$ of patients. ${ }^{26}$ Nevertheless, involvement of 2 or more sinuses was reported in $52 \%$ of patients. ${ }^{26}$ 
The transverse sinus was the most commonly affected sinus, which is similar to what is described in published literature. ${ }^{16,19,21,26,30}$ The sigmoid sinus was the next most commonly affected sinus, which is similar to findings by Katjazi et $\mathrm{al}^{19}$ and Duman et $\mathrm{al}^{26}$ but different from observations by Kardas et $\mathrm{al}^{31}$ and Tanislav et al. ${ }^{7}$ Superior sagittal sinus was the most commonly thrombosed sinus in studies from KSA by Daif et $\mathrm{al}^{4}$ and Algahtani et al, ${ }^{13}$ which was affected less commonly than the transverse and sigmoid sinuses in our study. Involvement of the internal jugular vein was higher in our study compared with international studies..$^{16,26}$

Intracerebral hemorrhage (ICH) can be observed in $15-49 \%$ of patients with CVT. ${ }^{32}$ Hemorrhagic venous infarction was detected in a higher number of patients in our study than reported by Duman et al, ${ }^{26}$ but nearly the same frequency has been reported previously. 19,29

In our study, etiology was undetermined in only $5.5 \%$ of patients, compared with $27 \%$ by Daif et al ${ }^{4}$ and $20 \%$ by Algahtani et al. ${ }^{8}$ Studies from KSA have found variation regarding pregnancy and puerperium as the predisposing factor: Algahtani et $\mathrm{al}^{8}$ identified it as the most common risk factor while Katjazi et $\mathrm{al}^{19}$ reported it in $4.5 \%$ of patients and Daif et $\mathrm{al}^{4}$ reported it in only one patient. Puerperium was the identified cause of CVT in $13.8 \%$ of our patients, which is somewhat less than the figure reported in a large multicenter study (18\%). ${ }^{25}$ Oral contraceptive pills usage was reported in $11 \%$ of our cases, which is similar to previously reported frequencies. ${ }^{12,21}$ Several studies have reported the usage of OCPs in nearly half of patients. ${ }^{13,18}$ Underlying prothrombotic disorder was found in $33.3 \%$ of our patients, which is higher than mentioned in some studies $\left(19.8 \%,{ }^{13} 26.4 \%,{ }^{16} 6.7 \%{ }^{26}\right)$ but in agreement with a study by Ferro et $\mathrm{al}^{14}(34 \%) .{ }^{27}$ We did not find protein $\mathrm{C}$ deficiency in any of our patients. Bechet's disease was found in $25 \%$ of cases by Daif et $\mathrm{al}_{1}{ }^{4} 9.4 \%$ by Duman et $\mathrm{al}^{24}$ and in none by Kajtazi et al. ${ }^{19}$ It was found in 2 of our patients, which is similar to a finding by Algahtani et $\mathrm{al}^{13}$ Infection as the etiology was less frequent than reported in another study from KSA. ${ }^{13}$

Among patients with CVT, only $1.6 \%$ of cases could be attributed to inflammatory bowel disease (IBD). ${ }^{33}$ Ulcerative colitis, one form of IBD, was found in 2 $(5.5 \%)$ of our cases and in one patient was the first presentation of disease. This is an important finding as it emphasizes that the diagnosis should not only be considered in any patient with IBD having neurological symptoms, but it must be considered among the multiple causes of CVT as well. Anemia is considered one of the important risk factors for CVT. The reported frequency of anemia was $3.2 \%$ by Duman et $\mathrm{al}^{26}$ and $9.2 \%$ Ferro et al. ${ }^{16} \mathrm{~A}$ case-control study reported a figure of $27 \%$ in patients with CVT compared with $6.5 \%$ in the control group $(p<0.001) .{ }^{34,36}$ This study detected a much higher frequency of anemia of $30 \%$, which is of significance as it can be prevented.

Recent advances in diagnostic and treatment modalities have improved outcomes in patients with CVT. The estimated mortality rate was approximately $5.6 \%$ in a meta-analysis by Dentali et al. ${ }^{35}$ The mortality rate in our study was $2.7 \%$. Other studies have reported mortality as $3.2 \%,{ }^{13} 4.3 \%,{ }^{16} 9.1 \%,{ }^{19}$ and $10 \% .{ }^{4}$ No mortality was observed by Duman et al ${ }^{24}$ related to CVT. Ferro et $\mathrm{al}^{16}$ have identified age $>37$ years, coma, male gender, intracranial hemorrhage, CNS infection, malignancy, and involvement of deep cerebral venous system as poor prognostic factors.

Study limitation. Small sample size is the major limitation of our study. It is a single-center study and therefore the results cannot be generalized to the whole population. A large, prospective multicenter study is needed to outline general characteristics of patients with CVT from KSA. There is a lack of uniformity in diagnostic neuroimaging modalities. Follow-up imaging was not available in all cases. Despite limitations, the clinical profiles and radiological features of patients admitted with CVT to our center have been described in detail.

In conclusion, the transverse sinus was the most frequently thrombosed sinus. Higher prevalence of iron deficiency anemia in patients with CVT is an important finding, which needs to be investigated further.

Acknowledgment. This research made use of the computational resources and technical services provided by the Scientific and High Performance Computing Center at Imam Abdulrahman Bin Faisal University, Dammam, Kingdom of Saudi Arabia. Also, the authors gratefully acknowledge Editage (www.editage.com) for English language editing.

\section{References}

1. Cutinho JM. Cerebral venous thrombosis. J Thromb Haemost 2015; 13 Suppl 1: S238-S244.

2. Bousser MG, Ferro JM. Cerebral venous thrombosis: an update. Lancet Neurol 2007; 6: 162-170.

3. Ghiasian M, Mansour M, Mazaheri S, Pirdehghan A. Thrombosis of the cerebral veins and sinuses in Hamadan, West of Iran. J Stroke Cerebrovasc Dis 2016; 25: 1313-1319.

4. Daif A, Awada A, Al-Rajeh S, Abduljabbar M, Al Tahan AR, Obeid T, et al. Cerebral venous thrombosis in adults: A study of 40 cases from Saudi Arabia. Stroke 1995; 26: 1193-1195.

5. Yaqub BA, Shamena AR, KolawoleTM, Patel PJ. Cerebrovascular disease in Saudi Arabia. Stroke 1991; 22: 1173-1176. 
6. Stam J. Thrombosis of cerebral veins and sinuses. N Engl J Med 2005; 352: 1791-1798.

7. Tanislav C, Siekmann R, Sieweke N, Allendörfer J, Pabst W, Kaps M, et al. Cerebral vein thrombosis: clinical manifestation and diagnosis. BMC Neurol 2011; 11: 69.

8. Algahtani HA, Aldarmahi AA. Cerebral venous sinus thrombosis. Neurosciences 2014; 19: 11-16.

9. Green M, Styles T, Russell T, Sada C, Jallow E, Stewart J, et al. Non-genetic and genetic risk factors for adult cerebral venous thrombosis. Thrombosis research 2018; 169: 15-22.

10. Narayan D, Kaul S, Ravishankar K, Suryaprabha T, Bandaru VS, Mridula KR, et al. Risk factors, clinical profile, and longterm outcome of 428 patients of cerebral sinus venous thrombosis: insights from Nizam's Institute Venous Stroke Registry, Hyderabad (India). Neurol India 2012; 60: 154-159.

11. Piazza G. Cerebral venous thrombosis. Circulation 2012; 125 : 1704-1709.

12. Fleet J, Birns J, Bhalla A. Cerebral venous thrombosis in adults. J Neurol Disord Stroke 2014; 2: 1033.

13. Algahtani HA, Abdu AP, Shami AM, Hassan AE, Madkour MA, Al-Ghamdi SM, et al. Cerebral venous sinus thrombosis in Saudi Arabia. Neurosciences 2011; 16: 329-334.

14. Ferro JM, Canhão P, Bousser MG, Stam J, Barinagarrementeria F, ISCVT Investigators. Cerebral vein and dural sinus thrombosis in elderly patients. Stroke 2005; 36: 1927-1932.

15. Zuurbier SM, Hiltunen S, Lindgren E, et al. Cerebral venous thrombosis in older patients. Stroke 2018; 49: 197-200.

16. Ferro JM, Canhão P, Stam J, et al., for the ISCVT Investigators. Prognosis of cerebral vein and dural sinus thrombosis: results of the International Study on Cerebral Vein and Dural Sinus Thrombosis (ISCVT). Stroke 2004; 35: 664-670.

17. Zuurbier SM, Arnold M, Middeldorp S, et al. Risk of cerebral venous thrombosis in obese women. JAMA Neurol 2016; 73: 579-584.

18. Misra UK, Kalita J, Chandra S, Kumar B, Bansal V. Low molecular weight heparin versus unfractionated heparin in cerebral venous sinus thrombosis: a randomized controlled trial. Eur J Neurol 2012; 19: 1030-1036.

19. Kajtazi NI, Zimmerman VA, Arulneyam JC, Al-Shami SY, Al-Senani FM. Cerebral venous thrombosis in Saudi Arabia Clinical variables, response to treatment, and outcome. Neurosciences 2009; 14: 349-354.

20. Dash D, Prasad K, Joseph L. Cerebral venous thrombosis: An Indian perspective. Neurol India 2015; 63: 318-328.

21. Rim HT, Jun HS, Ahn JH, Kim JH, Oh JK, Song JH, et al. Clinical Aspects of cerebral venous thrombosis: experiences in two institutions. J Cerebrovasc Endovasc Neurosurg 2016; 18 : 185-193.

22. Park DS, Moon CT, Chun YI, Koh YC, Kim HY, Roh HG. Clinical characteristics of cerebral venous thrombosis in a single center in Korea. J Korean Neurosurg Soc 2014; 56: 289-294.
23. Shahid R, Zafar A, Nazish S, Alsulaiman A, Alabdali M, Aljaafari $\mathrm{D}$, et al. Etiologic and clinical features of cerebral venous sinus thrombosis in Saudi Arabia. J Neurosci Rural Pract 2019; 10: 278-282.

24. Wasay M, Saadatnia M, Venketasubramanian N, Kaul S, Menon B, Gunaratne P, et al. Predictors of cerebral venous thrombosis and arterial ischemic stroke in Young Asian Women. J Stroke Cerebrovasc Dis 2011; 21: 689-694.

25. Ruiz-Sandoval JL, Chiquete E, Banuelos-Becerra LJ, TorresAnguiano C, González-Padilla C, et al. Cerebral venous thrombosis in a Mexican Multicenter Registry of Acute Cerebrovascular Disease: The RENAMEVASC Study. J Stroke Cerebrovasc Dis 2012; 21: 395-400.

26. Duman T, Uluduz D, Midi I, Bektas H, Kablan Y, Goksel BK, et al. A multicenter study of 1144 patients with cerebral venous thrombosis: the VENOST study. J Stroke Cerebrovasc Dis 2017; 26: 1848-1857.

27. Komro J, Findakly D. Cerebral Venous sinus thrombosis in adults with prothrombotic conditions: a systematic review and a case from our institution. Cureus 2020; 12: e7654.

28. Coutinho JM, Stam J. How to treat cerebral venous and sinus thrombosis. J Thromb Haemost 2010; 8: 877-883.

29. Breteau G, Mounier-Vehier E, Godefroy O, Gauvrit JY, Mackowiak-Cordoliani MA, Girot M, et al. Cerebral venous thrombosis 3-year clinical outcome in 55 consecutive patients. J Neurol 2003; 250: 29-35.

30. Wang JW, Li JP, Song YL, Tan K, Wang Y, Li T, et al. Clinical characteristics of cerebral venous sinus thrombosis. Neurosciences 2015; 20: 292-295.

21. Karadas S, Milanlioglu A, Gonullu H, Sayin R, Aydin MN. Cerebral venous sinus thrombosis presentation in emergency department in Van, Turkey. J Pak Med Assoc 2014; 64: 370-374.

32. de Bruijn SF, Stam J. Randomized, placebo-controlled trial of anticoagulant treatment with low-molecular weight heparin for cerebral sinus thrombosis. Stroke 1999; 30: 484-488.

33. Benavente L, Moris G. Neurologic disorders associated with inflammatory bowel disease. Eur J Neurol 2011; 18: 138-143.

34. Coutinho JM, Zuurbier SM, Gaartman AE, Dikstaal AA, Stam J, Middeldorp S, et al. Association between anemia and cerebral venous thrombosis: case-control study. Stroke 2015; 46: 2735-2740.

35. Dentali F, Crowther M, Ageno W. Thrombophilic abnormalities, oral contraceptives, and risk of cerebral vein thrombosis: A meta-analysis. Blood 2006; 107: 2766-2773.

36. Ulivi L, Squitieri M, Cohen H, Cowley P, Werring DJ. Cerebral venous thrombosis: a practical guide. Practical Neurol 2020; 20: 356-367. 